\title{
Silicon nanowires based resistors as gas sensors
}

\author{
F. Demami, L. Ni, R. Rogel, A. C. Salaun, L. Pichon \\ GM-IETR, Université de Rennes 1, 35000 Rennes, France
}

Silicon nanowires (SiNWs) are synthesized following two methods: i) the VLS (Vapor-LiquidSolid) growth technique (bottom up approach), and ii) the sidewall spacer fabrication (top down approach) commonly used in microelectronic industry. The VLS growth technique uses gold nanoparticles to activate the vapor deposition of the precursor gas and initiate $100 \mathrm{~nm}$ diameter SiNWs network growth. In the case of the sidewall spacer method, a polysilicon layer is deposited by LPCVD (Low Pressure Chemical Vapor Deposition) technique on $\mathrm{SiO}_{2}$ wall patterned by conventional UV lithography technique. Polysilicon film is then plasma etched. Accurate control of the etching rate leads to the formation of spacers with a $100 \mathrm{~nm}$ curvature radius that can be used as polysilicon NWs. Each kind of nanowires is integrated into resistors fabrication. Electrical measurements show the potential usefulness of these SiNWs based resistors as gas sensors for ammonia $\left(\mathrm{NH}_{3}\right)$ and smoke detection. 


\section{Introduction}

Semiconducting nanowires are currently attracting much attention as promising components for future nanoelectronic devices such as nanowire field effect transistors [1], photonic and optoelectronic devices [2], and more particularly as chemical or biological sensors [3-5]. The need of a fast and precise detection of early disease symptoms, as well as the need of environment safety, becomes now the main leitmotiv of the societal development. The incorporation of semiconducting nanowires into chemical and biological sensors applications receives a great interest. As their surface can be sensitive to charged species combined with their high surface to volume ratio, semiconducting nanowires are the subject of intense research activities for high sensitivity chemical sensor fabrication.

The first application of silicon nanowires (SiNWs) in biological and chemical sensors was reported by Lieber's group in 2001 [3]. As SiNWs synthesis can be compatible with the established Si technology, SiNWs based sensor integration will allow a lower manufacturing cost, in addition to the advantageous electronic features of embedded detection and signal processing in silicon technology. The intrinsic reliability of the well-known semiconductor CMOS (Complementary Metal Oxide Semiconductor) process also guarantees a reproducible and reliable diagnosis.

SiNWs can be prepared by the top-down approach, using various advanced methods such as ebeam [5], AFM [6] or deep UV [7] lithography. The main disadvantage of these advanced lithographic tools with nanometer size resolution rests on the high cost generated. An alternative way to synthesize SiNWs is the bottom-up approach that usually employs metal catalytic growth. The most commonly used growth technique is the VLS (Vapor-Liquid-Solid) method using metallic nanoparticles. The metal catalyst used is usually gold although other metals are also employed [8]. However, this approach suffers from the difficulty in precisely positioning the device. 
In this work, two types of non intentionally doped SiNWs are synthesized, without requiring costly lithographic tools, following the VLS growth technique (bottom up approach), and the sidewall spacer realization (top down approach) commonly used in microelectronic industry. These SiNWs are integrated into low temperature $\left(<600^{\circ} \mathrm{C}\right)$ silicon technology resistors fabrication and their ability as sensitive units for gas (ammonia) and ambiance (smoke) detections is demonstrated.

\section{Silicon nanowires synthesis and resistors fabrication}

\subsection{VLS SiNWs based devices}

The VLS mechanism was proposed in 1964 as an explanation for silicon whisker growth from the gas phase in the presence of a liquid gold droplet placed upon a silicon substrate [9]. Such mechanism is described following three steps: i) preparation of a liquid alloy droplet (catalyst) upon the substrate from which a wire is to be grown, ii) introduction of the substance to be grown as a vapor (precursor gas), which absorbs on the liquid surface, and diffuses into the droplet, and iii) supersaturation and nucleation at the liquid/solid interface leading to axial crystal growth.

Au-catalyst VLS SiNWs were synthesized and integrated into resistors. In this way, a heavily phosphorous in-situ doped amorphous silicon layer was first deposited by LPCVD (Low Pressure Chemical Vapor Deposition) technique at $550^{\circ} \mathrm{C}$ and $90 \mathrm{~Pa}$ on a substrate (silicon wafer or glass substrate) capped with a $\mathrm{SiO}_{2}$ buffer layer. Subsequent solid phase crystallization was performed at $600^{\circ} \mathrm{C}$ under vacuum to get a highly doped polycrystalline silicon (polysilicon) film. This film was patterned by Reactive Ion Etching (RIE) to define the geometry of the comb shape electrodes (interdigitated structure shown in Fig.1(a)). Au thin film $(\leq 5 \mathrm{~nm})$ was then deposited by thermal evaporation and locally removed using a lift off technique in order to define precise location for SiNWs growth (Fig.1(b)). High density Au-catalyst non intentionally doped VLS-SiNWs network was then synthesized by LPCVD technique at $460^{\circ} \mathrm{C}$ and $40 \mathrm{~Pa}$ using silane $\left(\mathrm{SiH}_{4}\right)$ as precursor 
gas. Due to the length of the SiNWs which can exceed $20 \mu \mathrm{m}$, bridges and contacts between SiNWs ensure the connection of these two heavily in-situ doped polysilicon islands leading to the formation of resistors in a 3D configuration (Fig.1(c)). This synthesis results in a tangled growth of $100 \mathrm{~nm}$ diameter SiNWs insuring electrical contact between the two doped electrodes (Fig.1(d)). LPCVD parameters were chosen in order to avoid silicon deposition on areas uncovered with Au thin film.

Previous study [10] of electrical behavior of such VLS SiNWs based resistors showed that the global electrical resistance results from the contribution of the silicon NWs. In addition, such electrical resistance value is both dependent on the length and the number of effective conductive paths [11] (difficult to estimate).

\subsection{Spacer method SiNWs based devices}

The sidewall spacer method is commonly used in submicron scale device silicon technology to insulate device active area. In our case, the spacer at nanometric scale made of silicon constitutes the nanowire. This method is an alternative way to synthesize SiNWs in a 2D configuration (Fig.2).

At first, a dielectric film A was deposited and patterned into islands by conventional UV lithography. Then, an undoped polysilicon layer was deposited by LPCVD technique. Silicon film was deposited in an amorphous state at $550^{\circ} \mathrm{C}$ and $90 \mathrm{~Pa}$, and then crystallized by thermal annealing under vacuum at $600^{\circ} \mathrm{C}$ during 12 hours. Accurate control of the polysilicon layer RIE rate leads to the formation of nanometric size sidewall spacers that can be used as nanowires (Fig 2(a)). The feasibility of this technological step was previously reported [12]. Thus, non intentionally doped polysilicon NWs with a $100 \mathrm{~nm}$ curvature radius were synthesized (see Fig.2(b)). This method allows the fabrication of parallel SiNWs network over a large area. Such polysilicon NWs were integrated into resistors devices in coplanar structure. In this case, contacts 
electrodes were made of thermally evaporated aluminum and defined by wet etching (Fig.2(c)). Finally a thermal annealing in forming gas $\left(\mathrm{N}_{2}: \mathrm{H}_{2}\right)$ was carried out to ensure good electrical contacts. Resistors were fabricated with $10 \mu \mathrm{m}$ length parallel SiNWs.

\section{Results and discussion}

\subsection{Methodology}

Performances of SiNWs based resistors are characterized measuring static electrical characteristics I-V using HP4155B semiconductor parameter analyzer. The resistance $(\mathrm{R}=\mathrm{V} / \mathrm{I})$ is deduced from the slope of the linear I-V curves for each device previously reported [11, 12]. Rvalues are then reported as function of time for devices under exposure to ammonia or smoke. Prior to measurements, devices were submitted to high diluted hydrofluoridric acid (2\%) aqueous solution to remove the native oxide on the SiNWs surface in order to promote chemical species adsorption. Then, SiNWs based devices were put into a vacuum chamber. The electrical measurements were carried out at room temperature with an all or nothing protocol: it means after evacuating the chamber $\left(\mathrm{P}=10^{-4} \mathrm{mbar}\right)$ ammonia (or burnt incense stick smoke) was introduced without any carrier gas. Ammonia partial pressure during SiNWs based resistors exposure is about 200 mbar.

The ammonia or smoke detector response, $S_{\mathrm{g}}$, is defined as:

$$
S_{g}=\frac{R_{g}-R}{R_{g}}=\frac{I-I_{g}}{I}
$$

where R (I) and $R_{g}\left(I_{g}\right)$ are the resistance (current) values for devices in vacuum and reactive ambient respectively. 


\subsection{Chemical species detection}

The chemical responses of these SiNWs based resistors to ammonia or incense smoke exposure are presented in function of exposure time in Fig.3 and 4 respectively. For each SiNWs based device deduced values of $S_{g}$ are summed up in Table 1.

Upon exposure to ammonia cycling tests (Fig.3), time dependence change of the electrical resistance is found to indicate a quasi reversible trend suggesting that SiNWs could be reusable after exposure. For the two types of non doped SiNWs based devices, electrical resistance decreases because ammonia species act as donor of electrons (reducing agents) at the SiNWs surface [13]. Under incense smoke exposure, the cycling tests show a decrease of the electrical resistance (Fig. 4), which shows that chemical species adsorbed at the SiNWs surface mostly act as reducing agents. However, the reversibility is not effective because it is lower after the second cycle. This may be related to the chemical species contained in the incense smoke. Indeed, the gas products from burning incense include $\mathrm{CO}, \mathrm{CO}_{2}, \mathrm{NO}_{2}, \mathrm{SO}_{2} \ldots$ The lower value observed for the two types of SiNWs based resistors after the second cycle suggests that some of these species might no be desorbed after exposure.

As the gas molecules adsorption occurs at the SiNWs surface, electrons are transferred to the SiNWs crystal core. Such interactions induce significant changes in the carriers transport along the nanowires and SiNWs electrical resistance in different possible cumulative ways. First, as the SiNWs conductance can be modulated by an applied voltage [13,14], positively charged gas molecules (electron donors) binded on SiNWs surface can modulate their conductance by changing the volume of the conductive layer. In this case, ammonia and incense smoke may act as chemical gates. In other words, it means that the Fermi level of the Si nanowires is shifted reducing the sample electrical resistance. This phenomenon may occur on the two SiNWs structures. Moreover, carrier transport strongly depends on structural nanowires defects. So, we have to consider effects of grain boundaries (spacer method - SiNWs) and contact resistance 
between nanowires (VLS method). Through charge exchange, gas molecules adsorbed may play a significant role in decreasing the potential barrier height at the tunnel junction of two crossed SiNWs (VLS method) or the potential barrier height at the grain boundaries between two grains (spacer method). Previous work reported such effect [15]. For the two types of SiNWs based devices, the electrical resistance decrease under ammonia or incense smoke exposure suggests that electrons transfer and potential barriers lowering effects may dominate. In such case, the higher measured relative responses for poly-SiNWs based resistors could be related to the higher defects density for the polysilicon SiNWs due to their polycrystalline texture, promoting a higher chemical species adsorption. However, the lower relative responses for VLS SiNWs based resistors could be explained by another reason: gold impurities embedded within NWs bulk during the process growth. Such impurities might act as recombination centers, and therefore they could be responsible of a lowering of the electrons transfer effect related to chemical species detection.

These first qualitative results show the potential usefulness of the two kinds of nanowires as sensitive units for high sensitive gas sensors applications operating at room temperature.

\section{Conclusion}

In summary, we report the SiNWs synthesis using bottom up or top down approaches, over a large area and with high density, without requiring costly lithographic tools. These nanowires, integrated into resistors electrically tested, show their sensitivity under exposure to ammonia and smoke due to ambient charged species detection. The first results serve as proof-of-concept for a new kind of SiNWs as promising sensitive units for high performance room temperature chemical sensors compatible with classical integrated silicon technology. Further work will focus on the SiNWs surface functionalization for sensing different kinds of gases as well as on the optimization of the surface/volume ratio to get high sensivity sensor. 


\section{Acknowledgments}

This work was supported by the Agence Nationale de la Recherche (France) with the Project Sena (ANR-09JCJC-0072-01).

\section{References}

[1] J. Goldberger, A. I. Hochbaum, R. Fan, P Yang, Silicon Vertically Integrated Nanowire Field Effect Transistors, Nanoletters, 6(5) (2006) 973

[2] C. Yang, C. J. Barrelet, F. Capasso, C.M. Lieber, Single p-Type/Intrinsic/n-Type Silicon Nanowires as Nanoscale Avalanche Photodetectors, Nanoletters, 6(12) (2006) 2929

[3] Y. Cui, Q. Wei, H. K. Park, C. M. Lieber, Nanowire nanosensors for highly sensitive and selective detection of biological and chemical species, Science, 293(5533) (2001) 1289.

[4] L.M. Lechuga J. Tamayo, M. Álvarez, L.G. Carrascosa, A. Yufera, R. Doldán, E. Peralías, A. Rueda, J.A. Plaza, K. Zinoviev, C Domínguez, A. Zaballos, M. Moreno, C. Martínez-A, D. Wenn, N. Harris, C. Bringer, V. Bardinal, T. Camps, C. Vergnenègre, C. Fontaine, V. Diaz, A. Bernad., A highly sensitive microsystem based on nanomechanical biosensors for genomics applications, Sensors and Actuators, B 118 (2006 ) 2

[5] Z. Li, Y. Chen, X. Li, T.I. Kamins, K. Nauka, R. S. Williams, Sequence-specific label-free DNA sensors based on silicon nanowires, Nanoletters, 4(2) (2004) 245

[6] I. Ionica, L. Montes, S. Ferraton, J. Zimmermann, L. Saminadayar,V. Bouchiat, Field effect and colomb blockage in silicon on insulator nanostructures fabricated by atomic force microscope Solid State Electronics, 49 (2005) 1497

[7] L. Yang, D. H. Lee, Y. H. Chen, C. Y. Chang, S. D. Liu, C. C. Huang, VLSI Symp. Tech. Dig, (2004) 196

[8] T.I. Kamins, R.S. Williams, Y. L. Chang, Y.A. Chang, Chemival vapor deposition of Si nanowire nucleated by $\mathrm{TiSi}_{2}$ island on $\mathrm{Si}$, Appl. Phys. Lett., 76 (2000) 562. 
[9] R. S. Wagner, W. C. Ellis, Vapor-liquid-solid mechanism of single crystal growth, Appl. Phys. Lett, 4(5) (1964) 89.

[10] L. Ni, F. Demami, R. Rogel, A. C. Salaun, L. Pichon, Fabrication and electrical characterization of silicon nanowires based resistors, Mat. Sc. Eng, 6 (2009) 012013

[11] F. Demami, L. Ni, R. Rogel, A. C. Salaun, L. Pichon, Silicon nanowires synthesis for chemical sensor applications, Procedia Engineering Eurosensors XXIV, Linz, Austria, September 5-8, 2010, pp. 351-354

[12] F. Demami, L. Pichon, R. Rogel, A. C. Salaun, Fabrication of polycrystalline silicon nanowires using conventional UV lithography, Mat. Sc. Eng, 6 (2009) 012014

[13] A. A. Talin, L. L. Hunter, F. Leonard, B. Rokad, Large area, dense silicon nanowire array chemical sensors, Appl. Phys. Lett, 89 (2006)153102

[14] J. Y. Yu, S. W. Chung, J. R. Heath, Silicon naowires: preparation, device fabrication, and transport properties, J. Phys. Chem, B 104 (2000) 11864.

[15] Y. Shimizu, M. Eg-ashira, Basic aspects and challenges of semiconductor gas sensors, MRS Bull, (June) (1999) pp. 18-24.

Demami Fouad received the B.E. degree in Telecommunications from the University of Sidi Mohamed Ben Abdellah, Fez (Morroco) in 2006 and the Master degree in Microelectronics and Microsystems from the University of Rennes (France) in 2007. Since 2007 he is Ph.D student at the Microelectronic Group of the Institute of Electronics and Telecommunications of Rennes (IETR). The subject of his research activities aims to silicon nanowires synthesis for electronic device applications.

Liang NI received the B.E. degree from the University of Shanghai in 2006 and the Master degree in micro- and nano- technologies from the University of Lille 1 (France) in 2008. Since 2008 he is 
$\mathrm{Ph} \mathrm{D}$ student at the Microelectronics Group, of Institute of Electronics and Telecommunication in Rennes (IETR), University of Rennes, (France). The subject of his research activities is in the field of silicon nanowires based sensors fabrication.

R. Rogel received his $\mathrm{PhD}$ degree in electronics in 2001 from university of Rennes. He is associate professor at the Microelectronic Group of the IETR (Institut d'Electronique et des Télécommunications de Rennes). His main research activities include the study of silicon based films deposited by LPCVD technique for electronic devices applications. His current research interests focus on silicon nanowires synthesis using top down and bottom approaches and their use as active area for the fabrication of silicon nanowires based devices. He is author and co-author of more than 20 international papers published in reviews or conference proceedings.

A. C. Salaun received the $\mathrm{Ph} \mathrm{D}$ degree in physics in 1996 and her Habilitation à Diriger des Recherches in 2010 from the university of Rennes (France). She is associate professor since 1998 at the Microelectronic Group of the IETR (Institut d'Electronique et des Télécommunications de Rennes), university of Rennes. Her activity is devoted to the micro-technology, with applications to chemical and biological sensors. She is author or co-author of 45 international papers published in reviews or conference proceedings.

L. Pichon received his Ph D degree in physics from the university of Rennes (France) in 1993, and his Habilitation à Diriger des Recherches in electronics from the university of Caen (France) in 2001. From 1996 to 2005 he was associate professor at the university of Caen. His research activities were focused on the polysilicon TFT fabrication and electrical characterization by low frequency noise measurements. Since 2005 he is professor at the Microelectronic Group of the IETR (Institut d'Electronique et des Télécommunications de Rennes-UMR CNRS 6164), 
university of Rennes. His research are focused on silicon nanowires technology for senors applications. He is author and co-author of 60 international papers published in reviews or conference proceedings. 


\section{Figure captions}

Figure 1: Key device fabrication steps of the SiNWs based resistors (VLS method). (a) Definition of the comb shape electrodes geometry on a $\mathrm{SiO}_{2}$ capped substrate. (b) Thin film $\mathrm{Au}$ local deposition after lift off technique. (c) Growth of Au-catalyst VLS SiNWs by LPCVD. (d) SEM top view observation of VLS SiNWs based resistor.

Figure 2: (a) Fabrication of polysilicon NWs by the sidewall spacer formation technique. (b) SEM pictures of $100 \mathrm{~nm}$ radius curvature polysilicon NWs cross section view. (c) Spacer method $10 \mu \mathrm{m}$ length polysilicon NW based resistor.

Figure 3: Electrical resistance variations versus time under ammonia exposure cycling test (a) VLS - SiNWs, (b) polysilicon nanowires (spacer method).

Figure 4: Electrical resistance variations versus time under smoke exposure cycling test (a) VLS SiNWs, (b) polysilicon nanowires (spacer method). 


\section{Table caption}

Table 1: Summary of the relative responses to ammonia and smoke for VLS and polysilicon SiNWs based resistors. 


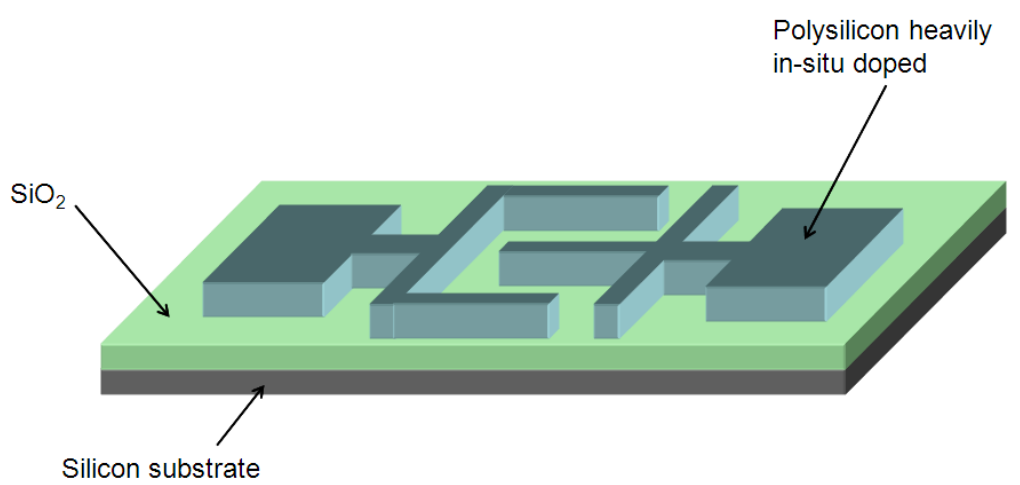

(a)

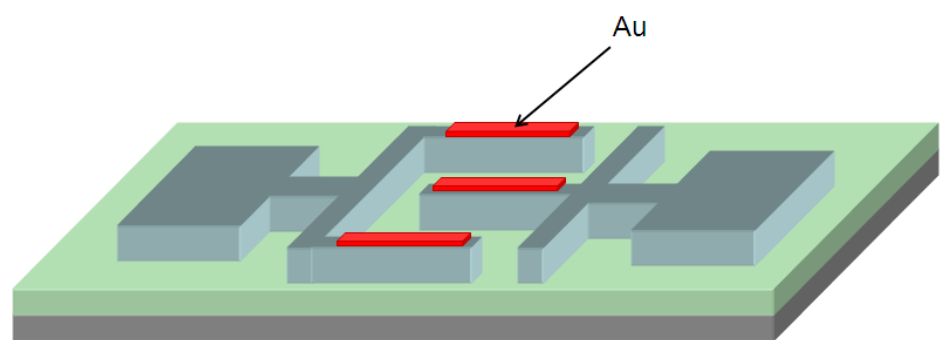

(b)

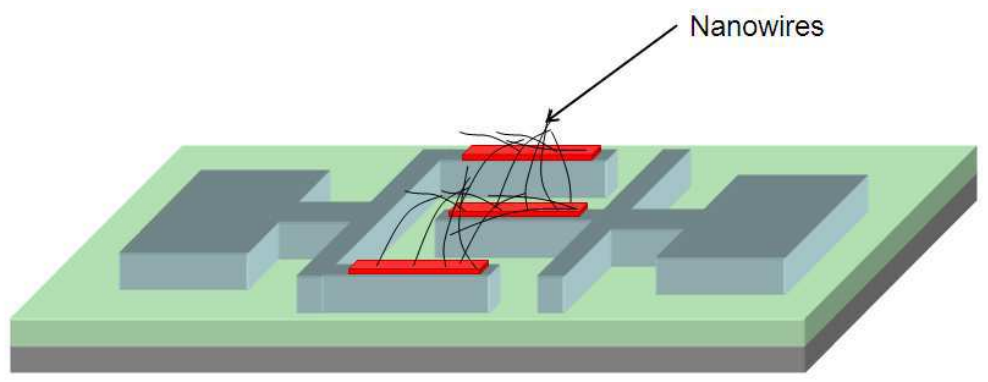

(c)

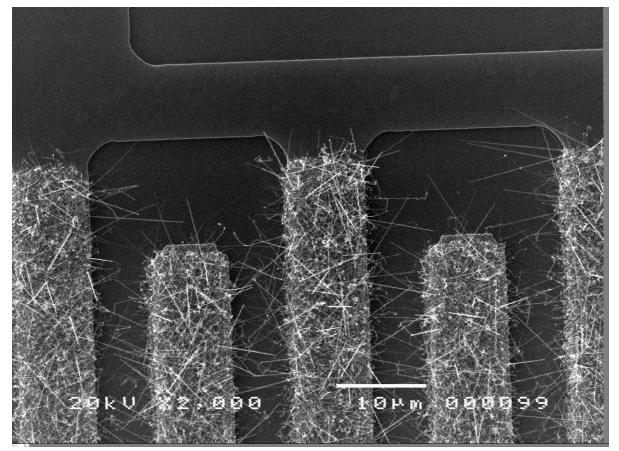

(d)

Figure 1 


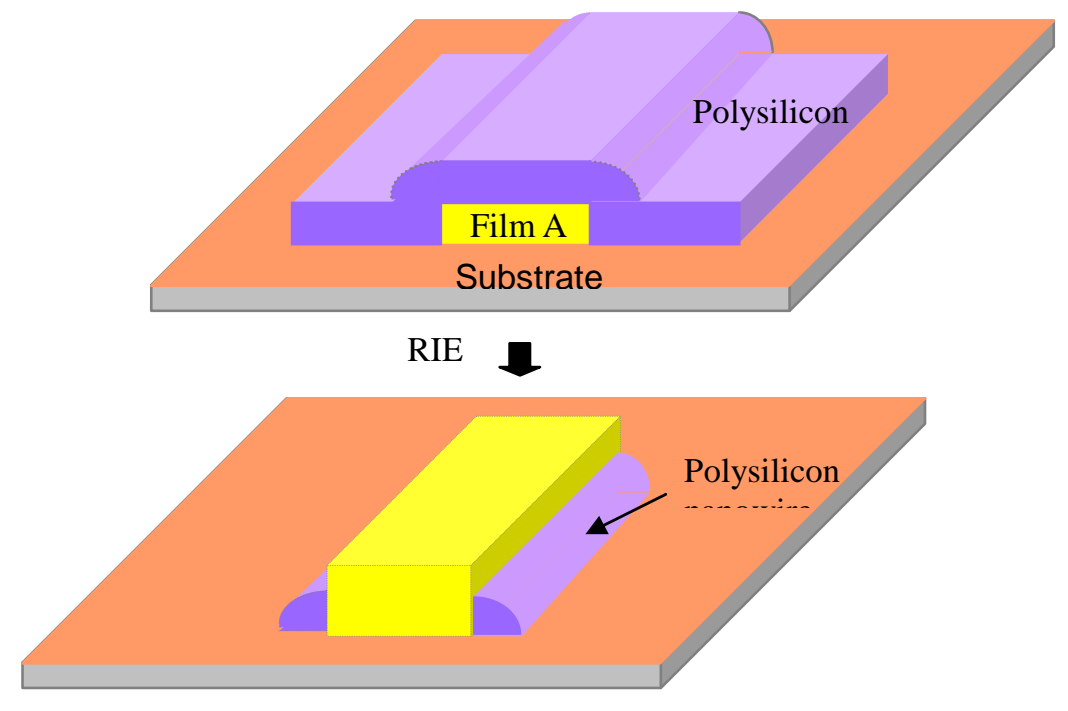

(a)

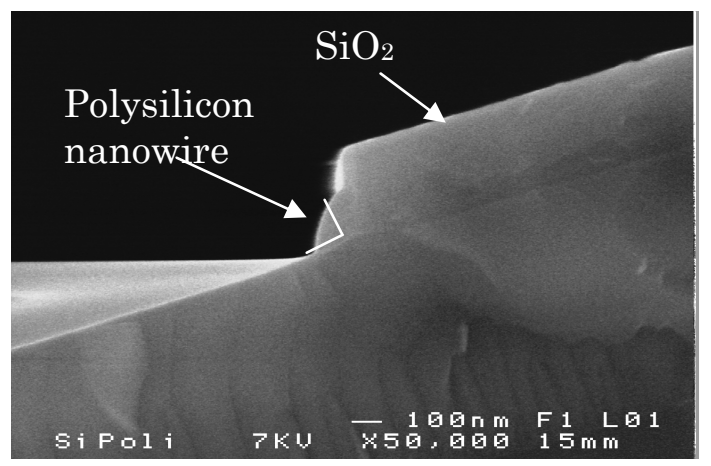

(b)

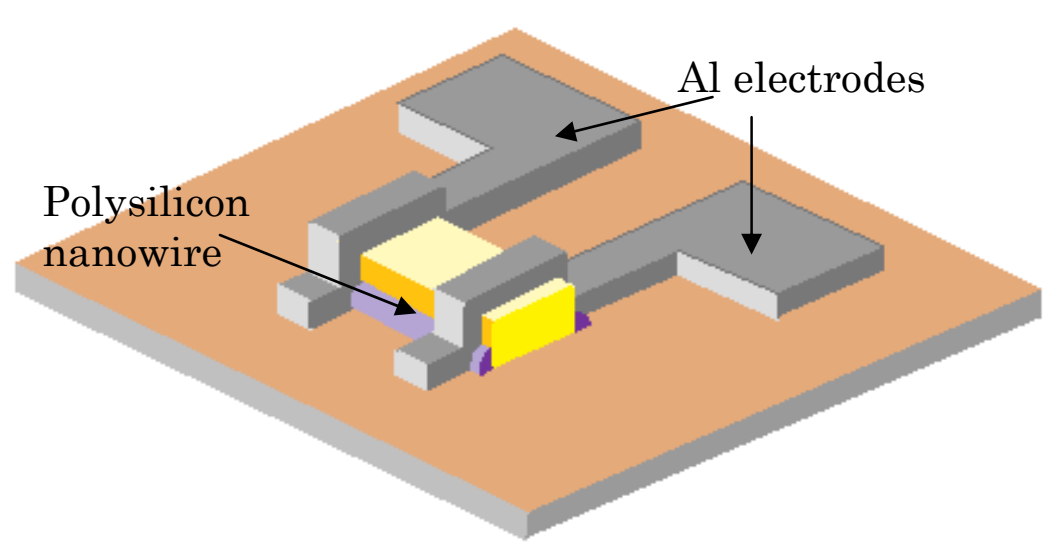

(c)

Figure 2 


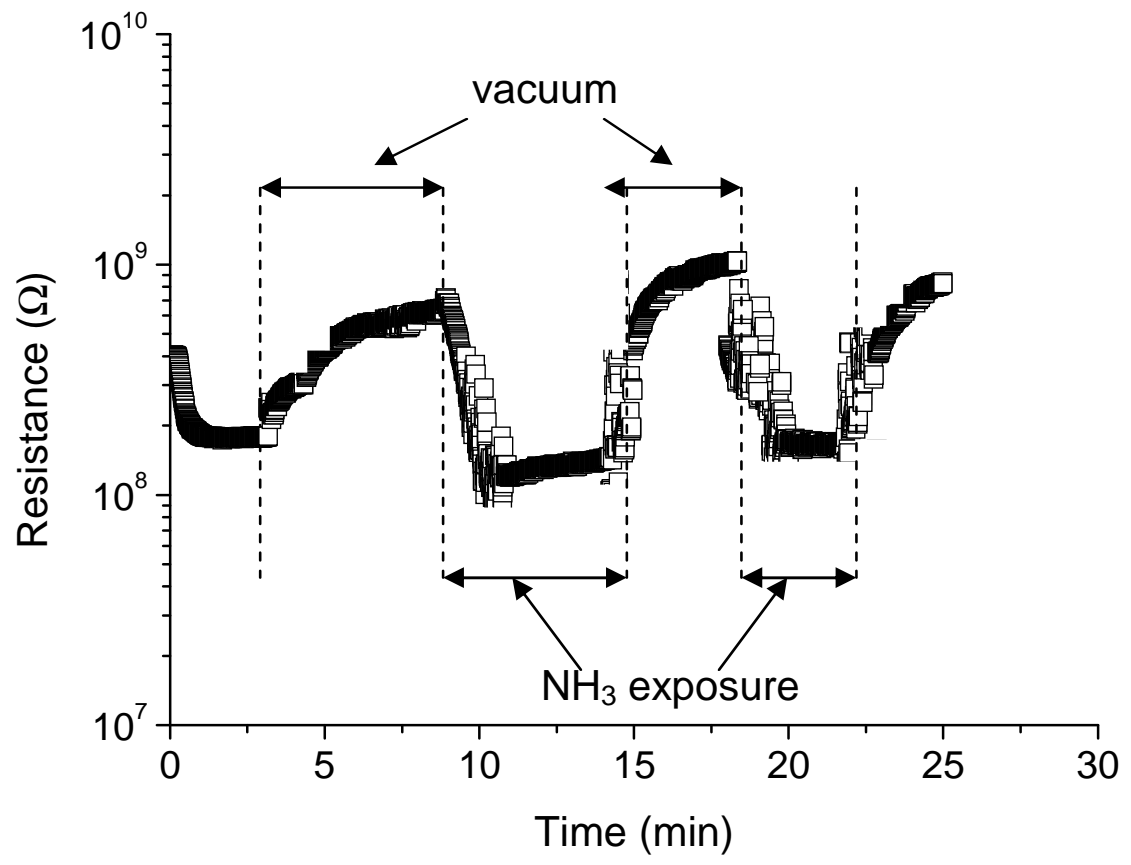

(a)

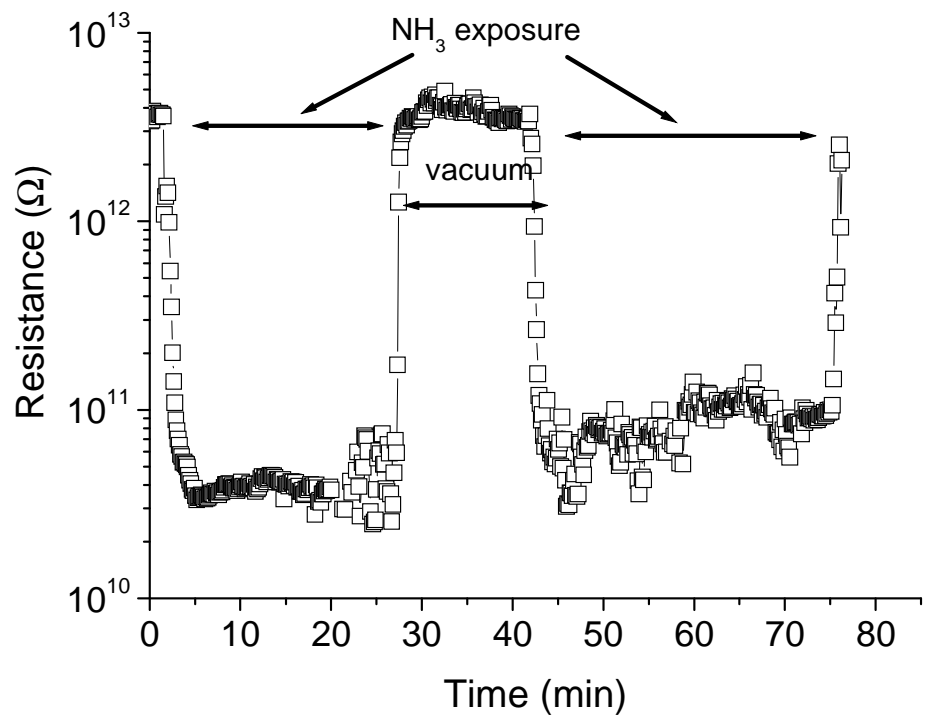

(b)

Figure 3 


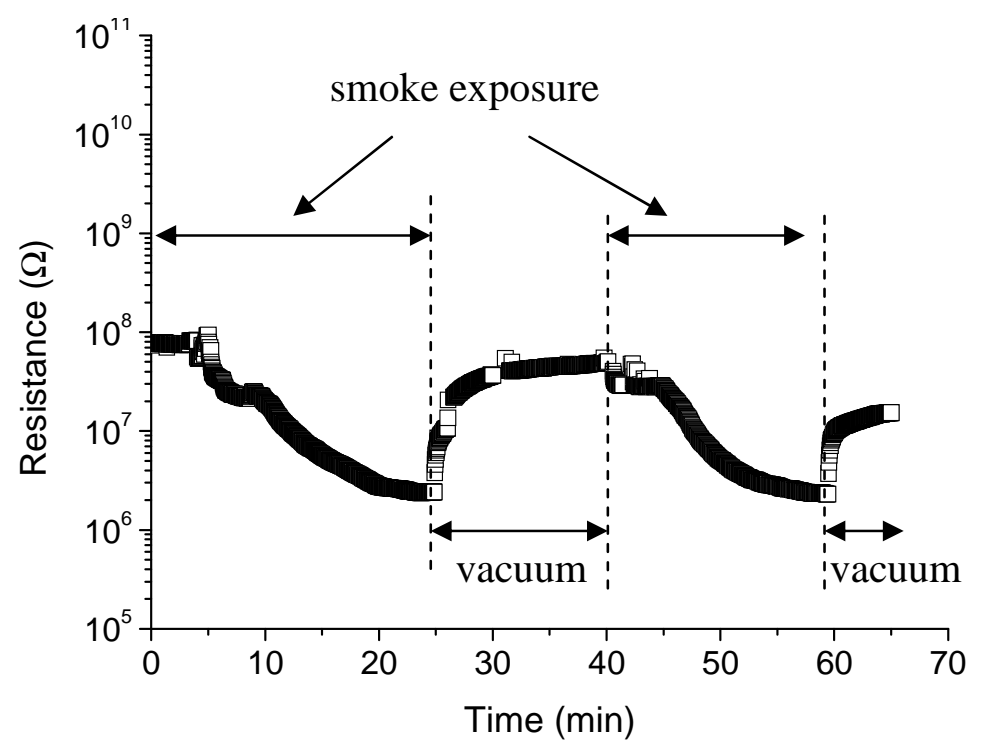

(a)

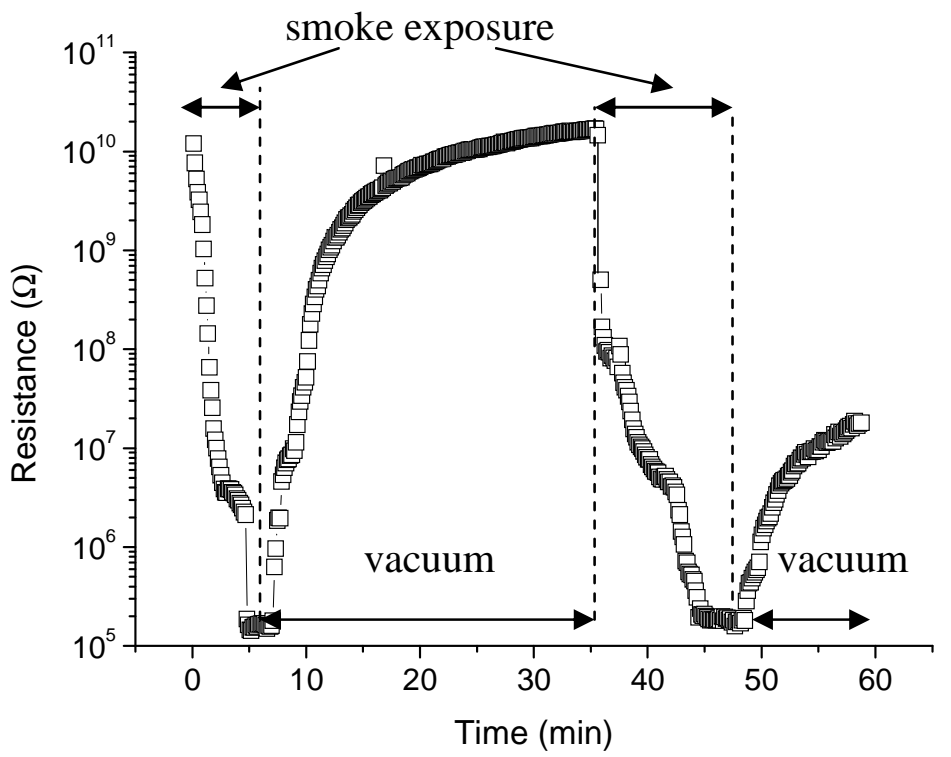

(b)

Figure 4 

$\mathrm{S}_{\mathrm{g}}(\%)$
$\mathrm{S}_{\mathrm{g}}(\%)$

$\left(\mathrm{NH}_{3}\right)$

(smoke)

VLS SiNWs based

$\sim 2000$

$\sim 2 \times 10^{4}$

resistors

Polysilicon SiNWs

$\sim 10^{4}$

$\sim 10^{7}$

based resistors

Table 1 